\title{
Function-valued adaptive dynamics and optimal control theory
}

Kalle Parvinen

Mikko Heino

Ulf Dieckmann (dieckmann@iiasa.ac.at)

\section{Approved by}

Pavel Kabat

Director General and Chief Executive Officer

February 2015 


\title{
Function-valued adaptive dynamics and optimal control theory
}

\author{
Kalle Parvinen ${ }^{1,4}$, Mikko Heino ${ }^{2,3,4}$ and Ulf Dieckmann ${ }^{4}$
}

Received: 1. Nov 2011 / Accepted: 7. May 2012

1. Department of Mathematics and Statistics, FI-20014 University of Turku, Finland

Tel.: +358-2-3335623, Fax: +358-2-231 0311, kalparvi@utu.fi

2. Department of Biology, University of Bergen, Box 7803, N-5020 Bergen, Norway

3. Institute of Marine Research, Box 1870 Nordnes, N-5817 Bergen, Norway

4. Evolution and Ecology Program, International Institute for Applied Systems Analysis, A-2361 Laxenburg, Austria

Appeared as: Journal of Mathematical Biology 67, 509-533

DOI:10.1007/s00285-012-0549-2 


\begin{abstract}
In this article we further develop the theory of adaptive dynamics of function-valued traits. Previous work has concentrated on models for which invasion fitness can be written as an integral in which the integrand for each argument value is a function of the strategy value at that argument value only. For this type of models of direct effect, singular strategies can be found using the calculus of variations, with singular strategies needing to satisfy Euler's equation with environmental feedback. In a broader, more mechanistically oriented class of models, the function-valued strategy affects a process described by differential equations, and fitness can be expressed as an integral in which the integrand for each argument value depends both on the strategy and on process variables at that argument value. In general, the calculus of variations cannot help analyzing this much broader class of models. Here we explain how to find singular strategies in this class of process-mediated models using optimal control theory. In particular, we show that singular strategies need to satisfy Pontryagin's maximum principle with environmental feedback. We demonstrate the utility of this approach by studying the evolution of strategies determining seasonal flowering schedules.
\end{abstract}

Keywords: adaptive dynamics, function-valued traits, theory of optimal control, Pontryagin's maximum principle, environmental feedback

AMS classification:92D15, 49-00

\title{
1 Introduction
}

Adaptive dynamics (Metz et al., 1992, 1996; Dieckmann and Law, 1996; Geritz et al., 1997, 1998) was originally formulated for scalar strategies $s \in \mathbb{R}$. Dieckmann et al. (2006) and Parvinen et al. (2006) presented theory for the adaptive dynamics of function-valued traits, i.e., strategies of the form $s(a) \in \mathbb{R}$ for $a \in \mathbb{R}$. In addition to developing this theory, Dieckmann et al. (2006) studied the evolution of metabolic investment strategies and the evolution of seasonal flowering schedules. Parvinen et al. (2006) further investigated models of metabolic investment strategies. The adaptive dynamics of function-valued strategies has been examined in various other applications, including maturation reaction norms (Ernande et al., 2004), foraging strategies on resource gradients (Heino et al., 2008), condition-dependent dispersal (Gyllenberg et al., 2008), timing of reproduction (Eskola, 2009; Eskola et al., 2011), size-dependent flowering (Rees and Ellner, 2009), and age-dependent sex-reversal (Calsina and Ripoll, 2010). This illustrates the wide applicability of function-valued traits to questions in evolutionary ecology. 
Dieckmann et al. (2006) introduced the canonical equation of functionvalued adaptive dynamics, which describes the expected rate of evolutionary change in a function-valued strategy. This canonical equation can be used to study the transient dynamics and convergence towards evolutionarily singular strategies (see, e.g., Heino et al., 2008). Already for vector-valued strategies, conditions for such convergence are more complicated than for scalar strategies (Christiansen, 1991; Marrow et al., 1996; Matessi and Di Pasquale, 1996; Geritz et al., 1998; Leimar, 2001; Meszéna et al., 2001), usually requiring dynamical analysis of the kind the canonical equation allows. In some simple cases, the equilibria of the canonical equation can be solved analytically, and singular strategies thus can be obtained. For using the canonical equation, the selection gradient needs to be calculated. The complexity of a model determines how difficult that calculation is.

Alternatively, for single-species evolution, singular strategies can be found by using the fact that they are local maxima or minima of a fitness function. Therefore, it is natural that the optimization method provided by the calculus of variations is a useful tool for finding singular strategies of function-valued traits and, under some circumstances, for determining their evolutionary stability (Parvinen et al., 2006). In particular, the calculus of variations is applicable when the invasion fitness (Metz et al., 1992) of a mutant with strategy $s_{\text {mut }}$ in the environment $E_{\text {res }}$ created by the resident population can be written as an integral of the form

$$
r\left(s_{\mathrm{mut}}, E_{\mathrm{res}}\right)=\int_{a_{\mathrm{min}}}^{a_{\max }} F\left(a, s_{\mathrm{mut}}(a), s_{\mathrm{mut}}^{\prime}(a), E_{\mathrm{res}}\right) \mathrm{d} a,
$$

where $s_{\text {mut }}(a)$ is defined for $a_{\text {min }} \leqslant a \leqslant a_{\max }$. Analogous methods, as described in section 7 of Parvinen et al. (2006), can be used in more complex cases, in which the invasion fitness $r\left(s_{\text {mut }}, E_{\text {res }}\right)=Z\left(I_{1}, I_{2}, \ldots, I_{n}\right)$ is a function of several integrals $I_{j}=\int_{a_{\min }}^{a_{\max }} F_{j}\left(a, s_{\text {mut }}(a), s_{\text {mut }}^{\prime}(a), E_{\text {res }}\right) \mathrm{d} a$ with $j=1, \ldots, n$. The main result of Parvinen et al. (2006) is that singular strategies in models of type (1) need to satisfy Euler's equation with environmental feedback. See, e.g., Heino et al. (2008) and Gyllenberg et al. (2008) for applications. In some special cases, the integrand $F\left(a, s_{\text {mut }}(a), s_{\text {mut }}^{\prime}(a), E_{\text {res }}\right)$ does not depend on $s_{\text {mut }}^{\prime}(a)$ and is a linear function of the strategy value $s_{\text {mut }}(a)$ of the mutant, so the resultant Euler's equation does not depend on $s_{\text {mut }}$. Finding singular strategies may then appear problematic, because $s_{\text {mut }}$ cannot be solved from an equation in which it does not appear. Instead, one obviously needs to find a resident environment $E_{\text {res }}$ such that Euler's equation is satisfied for any $s_{\text {mut }}$, for which model-specific methods may be needed (Eskola, 2009; Calsina and Ripoll, 2010). From an ecological point of view, the presence in equation (1) of the derivative $s_{\text {mut }}^{\prime}(a)=\frac{\mathrm{d}}{\mathrm{d} a} s_{\text {mut }}(a)$ may 
seem puzzling at first sight. Many problems of such type arise in physics, for example when $s(a)$ describes the position and $s^{\prime}(a)$ the velocity of an object. Also in evolutionary ecology, fitness functions of this form may naturally arise, albeit much more rarely, such as in the metabolic investment model of Parvinen et al. (2006).

The main purpose of this article is to present methods for analysing the evolution of function-valued traits in a broader class of models than those in which fitness is of form (1). We call this broader class of models processmediated models. In this more mechanistically-oriented class of models, the function-valued strategy affects a process described by ordinary differential equations

$$
\frac{\mathrm{d}}{\mathrm{d} a} y_{i}(a)=f_{i}\left(a, y_{1}(a), \ldots, y_{n}(a), s_{\mathrm{mut}}(a), E_{\mathrm{res}}\right),
$$

with state variables $y_{i}(a)$ for $i=1, \ldots, n$. The invasion fitness of a mutant in the environment $E_{\text {res }}$ created by the resident population is then assumed to be of the form

$$
r\left(s_{\mathrm{mut}}, E_{\mathrm{res}}\right)=\int_{a_{\min }}^{a_{\max }} F\left(a, y_{1}(a), \ldots, y_{n}(a), s_{\mathrm{mut}}(a), E_{\mathrm{res}}\right) \mathrm{d} a .
$$

In contrast, we refer to the simpler models of form (1) as direct-effect models.

For process-mediated models, the task of finding singular strategies becomes a problem of optimal control theory. The theory of optimal control has been used in the context of function-valued traits in game theory (Hamelin and Lewis, 2010), in quantitative genetics (Gomulkiewicz and Kirkpatrick, 1992; Gomulkiewicz and Beder, 1996; Beder and Gomulkiewicz, 1998; Jaffrézic and Pletcher, 2000; Kingsolver et al., 2001), and in life-history theory (Perrin and Sibly, 1993; Gilchrist et al., 2006). The novel feature considered in this article is the extension of the methods of optimal control theory to problems with environmental feedback, which is essential for tackling biologically realistic models.

Below we show that singular strategies in process-mediated models (2) need to satisfy Pontryagin's maximum principle (Pontryagin et al., 1962) with environmental feedback (section 2.2). To facilitate future applications, we present two worked-out examples by extending the seasonal flowering model of Dieckmann et al. (2006). In the first process-mediated extension (section 5), plants have a limited amount of resources that they can allocate to flower production at different times of the season. We illustrate that also in process-mediated models, a monomorphic singular function-valued strategy can be invadable by mutants, which can result in its evolutionary branching (Dieckmann et al., 2006). In the second process-mediated extension (section 6), plants split their allocation of the incoming energy flow between the 
growth of leaves and flowers. Both examples have been chosen such that the singular strategies can alternatively be found also based on the canonical equation: in this way, we can show how much more straightforward the analysis becomes by using the theory of optimal control.

\section{Theory for direct-effect and process-mediated models}

Following Metz et al. (1992), let $r\left(s_{\text {mut }}, E_{\text {res }}\right)$ denote the invasion fitness of a mutant, i.e., the exponential growth rate of a rare mutant with strategy $s_{\text {mut }}$ in the environment $E_{\text {res }}$ created by the resident. Let $S$ denote the set of possible strategies, often called the strategy space. According to Dieckmann et al. (2006), the following equation, referred to as the canonical equation of function-valued adaptive dynamics, describes the rate $\frac{\mathrm{d}}{\mathrm{d} t} s$ of expected evolutionary change in a function-valued strategy $s \in S$,

$$
\frac{\mathrm{d}}{\mathrm{d} t} s(a)=\frac{1}{2} \mu(s) \bar{n}(s) \int \sigma^{2}\left(a^{\prime}, a\right) G\left(a^{\prime}\right) \mathrm{d} a^{\prime}
$$

where $a$ is the argument of the function $s, \mu(s)$ is the mutation probability of trait $s, \bar{n}(s)$ is the trait-dependent equilibrium population size of the evolving population, $\sigma^{2}$ is the variance-covariance function of the mutation distribution, and $G$ is the selection gradient

$$
G(a)=\left.\frac{\mathrm{d}}{\mathrm{d} \epsilon} r\left(s+\epsilon \delta_{a}\right)\right|_{\epsilon=0}=\lim _{\Delta s \rightarrow \delta_{a}}\left[\left.\frac{\mathrm{d}}{\mathrm{d} \epsilon} r(s+\epsilon \Delta s)\right|_{\epsilon=0}\right] .
$$

The second equality specifies that the differentiation with respect to $\epsilon$ is to be done before the disturbance function $\Delta s$ is let to approach the Dirac delta function $\delta_{a}$, where $\delta_{a}\left(a^{\prime}\right)=\delta\left(a^{\prime}-a\right)$.

Obviously, strategies for which the selection gradient vanishes, $G(a)=0$ for all $a$, are equilibria of the canonical equation (3), and are called evolutionarily singular strategies. More in general, evolutionarily singular strategies are defined as strategies satisfying

$$
\left.\frac{\mathrm{d}}{\mathrm{d} \epsilon} r(s+\epsilon \Delta s)\right|_{\epsilon=0}=0
$$

for all $\Delta s$ for which $s+\epsilon \Delta s \in S$ when $|\epsilon|$ is sufficiently small.

As mentioned above, model complexity determines how easy (or difficult) calculating the selection gradient is. Next we discuss applicable methods for 
the evolutionary analysis of function-valued traits in two classes of models. First, we review the methods for direct-effect models, for which the calculation of the selection gradient is relatively easy, and singular strategies can be found using the calculus of variations; this summarizes the theory presented in Parvinen et al. (2006). Second, we extend the methods to process-mediated models, for which there is no general simplifying rule for calculating the selection gradient. We show how, instead, the theory of optimal control can be used to find singular strategies in such models.

\subsection{Direct-effect models and the calculus of variations}

Let us first consider models of function-valued traits $s(a)$, with $a_{\min } \leqslant a \leqslant$ $a_{\max }$, for which a mutant's fitness can be written as an integral of form (1). As shown by Parvinen et al. (2006, their equation 63), the selection gradient $G(a)$ for $a_{\min }<a<a_{\max }$ in this case becomes

$$
G(a)=F_{s}\left(a, s(a), s^{\prime}(a), E_{\mathrm{res}}\right)-\frac{\mathrm{d}}{\mathrm{d} a} F_{s^{\prime}}\left(a, s(a), s^{\prime}(a), E_{\mathrm{res}}\right),
$$

where the subscripts denote partial derivatives,

$$
\begin{gathered}
F_{s}\left(a, s(a), s^{\prime}(a), E_{\mathrm{res}}\right)=\frac{\partial}{\partial s(a)} F\left(a, s(a), s^{\prime}(a), E_{\mathrm{res}}\right), \\
F_{s^{\prime}}\left(a, s(a), s^{\prime}(a), E_{\mathrm{res}}\right)=\frac{\partial}{\partial s^{\prime}(a)} F\left(a, s(a), s^{\prime}(a), E_{\mathrm{res}}\right) .
\end{gathered}
$$

When there are no constraints on the strategy, and the strategy space $S$ is thus the set of all piecewise continuous functions $s:\left[a_{\min }, a_{\max }\right] \rightarrow$ $\mathbb{R}$, evolutionarily singular strategies are strategies $s$ for which the selection gradient vanishes, $G(a)=0$. According to (6), this condition is given by complementing the Euler's equation of the calculus of variations with the condition for ecological equilibrium under environmental feedback (Parvinen et al., 2006),

$$
\left\{\begin{array}{l}
\frac{\mathrm{d}}{\mathrm{d} a} F_{s^{\prime}}\left(a, s(a), s^{\prime}(a), E\right)=F_{s}\left(a, s(a), s^{\prime}(a), E\right), \\
r(s, E)=0 .
\end{array}\right.
$$

See Parvinen et al. (2006) for a more detailed description of the use of the calculus of variations for finding singular strategies. Here we just discuss the selection gradient and conditions for singular strategies at boundaries $a_{\text {min }}$ and $a_{\max }$, because these require special attention when $F$ depends on the derivative $s^{\prime}(a)$.

When $F$ does not depend on the derivative $s^{\prime}(a)$, the selection gradient (6) reduces to $G(a)=F_{s}(a, s(a))$ (Dieckmann et al., 2006, their equation 3c). 
This equality is valid for all relevant arguments $a, a_{\min } \leqslant a \leqslant a_{\max }$, including the boundaries, which thus require no special treatment. However, as we show in Appendix A, for a dependence of $F$ on $s^{\prime}(a)$, the selection gradient at the boundaries is infinite, unless $F_{s^{\prime}}=0$ at $a_{\min }$ and $a_{\max }$. Therefore, the selection pressure at the boundaries is infinitely stronger than elsewhere, keeping $F_{s^{\prime}}=0$ at the boundaries at all times.

Considering the other components of the selection gradient derived by Dieckmann et al. (2006), the mutation probability $\mu(s)$ and the equilibrium population size $\bar{n}(s)$ require no special treatment compared with the case without the $s^{\prime}$ dependence. It is also reasonable to assume that the derivative $s^{\prime}$ has no effect on the variance-covariance function $\sigma^{2}$ of the mutation distribution. We thus obtain the following result:

Proposition 1. If the fitness function is of form (1), the selection gradient (4) for $a_{\min }<a<a_{\max }$ can be written as (6). For the canonical equation (3), we obtain the boundary condition $F_{s^{\prime}}=0$ at $a_{\min }$ and $a_{\max }$.

Proof. The derivation of equation (6) was already given by Parvinen et al. (2006). In Appendix A we repeat this proof of the first part of Proposition 1 and then complement it by proving the second part of Proposition 1.

While earlier work had already shown that $F_{s^{\prime}}=0$ at the boundaries has to be satisfied for singular strategies, i.e., for potential evolutionary outcomes, Proposition 1 makes it clear that it has to hold for evolutionary transients.

We study an example of this type of model in section 4 , by extending the seasonal flowering model of Dieckmann et al. (2006). In that model, flowers opening at different times of the season experience different conditions, such as different probabilities of setting seed. It is therefore natural to study the flowering intensity as a function-valued trait, in which case fitness can be expressed as an integral of form (1).

A more mechanistic approach results from assuming that a plant's flowering intensity is determined by various processes, such as the use of energy reserves for flower production at different times of the season, the timedependent resource allocation between leaf growth and flower production, and the loss of flowers due to dropping or pests. This requires studying a more general class of models, which we consider next.

\subsection{Process-mediated models and the theory of opti- mal control}

Let us now consider the class of models (2) described by state variables $y_{i}(a)$ for $i=1, \ldots, n$ and by a function-valued strategy $s(a)$ with $a_{\min } \leqslant a \leqslant a_{\max }$. 
We assume that the functions $y_{i}(a)$ satisfy the following system of ordinary differential equations,

$$
\frac{\mathrm{d}}{\mathrm{d} a} y_{i}(a)=f_{i}\left(a, y_{1}(a), \ldots, y_{n}(a), s(a), E_{\mathrm{res}}\right),
$$

and that the invasion fitness of a mutant with strategy $s_{\text {mut }}$ in the environment $E_{\text {res }}$ created by the resident can be expressed as

$$
r\left(s_{\mathrm{mut}}, E_{\mathrm{res}}\right)=\int_{a_{\min }}^{a_{\max }} F\left(a, y_{1}(a), \ldots, y_{n}(a), s_{\mathrm{mut}}(a), E_{\mathrm{res}}\right) \mathrm{d} a .
$$

In order to determine the selection gradient, we need to calculate the derivative of $r\left(s+\epsilon \Delta s, E_{\mathrm{res}}\right)$ with respect to $\epsilon$. Since the state variables $y_{i}(a)$ depend on the strategy, and thus on $\epsilon$, this derivative does not yield as simple an expression as for the previous class of models. Sections 5.1 and 6.1 nevertheless show examples of the model-specific calculation of such selection gradients. For determining selection gradients in process-mediated models, no general method exists.

For process-mediated models, the task of finding evolutionarily stable strategies (Maynard Smith, 1976, 1982), or more in general, evolutionarily singular strategies, is closely related to problems of optimal control. As in models of form (1), singular strategies cannot be found through the straightforward application of a standard optimization method because of the environmental feedback. More specifically, the task is to find a strategy $s$ which creates the environment $E_{\text {res }}$ such that $s$ solves the problem of optimal control given by (9) and (10). In other words, such singular strategies need to satisfy Pontryagin's maximum principle (Theorem 2) with environmental feedback, $r(s, E)=0$.

For the sake of completeness, we repeat the corresponding theorem below. While the original theorem was presented for a minimization problem, here we have formulated it for a maximization problem, because evolutionarily stable strategies are local maxima of invasion fitness. The proof in its full form is rather lengthy; details can be found in Pontryagin et al. (1962) or in textbooks on optimal control theory.

Theorem 2 (Pontryagin's maximum principle). If $s(a)$ is a piecewise smooth optimal control function maximizing

$$
r(s, E)=\Psi\left(\mathbf{y}\left(a_{\max }\right), E\right)+\int_{a_{\min }}^{a_{\max }} F(a, \mathbf{y}(a), s(a), E) d a
$$


with $\mathbf{y}(t)=\left\{y_{1}(t), \ldots, y_{n}(t)\right\}$ and

$$
\begin{aligned}
\frac{d}{d a} y_{i}(a) & =f_{i}(a, \mathbf{y}(a), s(a), E) \text { for } i=1, \ldots, n, \\
y_{i}\left(a_{\min }\right) & =y_{0, i} \quad \text { for } i=1, \ldots, n, \\
y_{i}\left(a_{\max }\right) & =\hat{y}_{i} \quad \text { for } i \in N \subset\{1, \ldots, n\},
\end{aligned}
$$

then there exists a nonzero vector $\lambda(a)$, that satisfies the conditions

$$
\begin{aligned}
\frac{d}{d a} \lambda_{i}(a) & =-H_{y_{i}} \quad \text { for } i=1, \ldots, n, \\
\lambda_{i}\left(a_{\max }\right) & =\Psi_{y_{i}}\left(\mathbf{y}\left(a_{\max }\right), E\right) \quad \text { for } i \notin N,
\end{aligned}
$$

where

$$
\begin{aligned}
& H(a, \mathbf{y}(a), s(a), \lambda(a), E) \\
& \quad=F(a, \mathbf{y}(a), s(a), E)+\sum_{i=1}^{n} \lambda_{i}(a) f_{i}(a, \mathbf{y}(a), s(a), E)
\end{aligned}
$$

is called the Hamiltonian function, which is maximized by $s(a) H$ for each a,

$$
\begin{aligned}
& H(a, \mathbf{y}(a), s(a), \lambda(a), E) \\
& \quad=\min _{\hat{s}(a)} H(a, \mathbf{y}(a), \hat{s}(a), \lambda(a), E) \text { for all } a \in\left[a_{\min }, a_{\max }\right] .
\end{aligned}
$$

Corollary A necessary condition for a strategy s to be evolutionarily stable is that it satisfies the conditions for a piecewise smooth optimal control function maximizing (11) together with $r(s, E)=0$.

For the special case $n=1$ with $y_{1}^{\prime}(a)=f_{1}\left(a, y_{1}(a), s(a)\right)=s(a)$ the optimal control problem (11) can be solved by the calculus of variations. If there are no constraints on the optimal control function $s$, the maximization of the Hamiltonian means that

$$
0=H_{s}\left(a, y_{1}(a), s(a), \lambda_{1}(a)\right)=F_{s}(a)+\lambda_{1}(a)
$$

and thus $\lambda_{1}(a)=-F_{s}(a)$. Substituting that into the differential equation for $\lambda_{1}$ yields

$$
\frac{\mathrm{d}}{\mathrm{d} a} F_{s}(a)=F_{y_{1}}(a)
$$

which recovers Euler's equation. Therefore, for direct-effect models (1) with unconstrained strategies, approaches alternatively based on the calculus of variations or on the theory of optimal control (Theorem 2) are equivalent.

To explain and demonstrate the utility of optimal control theory for analyzing the adaptive dynamics of function-valued traits is the main purpose of this article. For illustration, we apply the resultant methods to extensions of the seasonal flowering model by Dieckmann et al. (2006). The conclusion of this section on theory is the following table of available methods: 
Table 1: Description of methods applicable to different model classes

\begin{tabular}{|l|l|l|}
\hline & Direct-effect models & Process-mediated models \\
$r(s, E)=$ & $\int_{a_{\min }}^{a_{\max }} F\left(a, s(a), s^{\prime}(a), E\right) \mathrm{d} a$ & $\begin{array}{l}\int_{a_{\min }}^{a_{\max }} F(a, \mathbf{y}(a), s(a), E) \mathrm{d} d \\
\text { with state variables } y_{i} \\
\text { satisfying } \frac{\mathrm{d}}{\mathrm{d} a} y_{i}(a) \quad= \\
f_{i}(a, \mathbf{y}(a), s(a), E)\end{array}$ \\
\hline $\begin{array}{l}\text { Method } \\
\text { for } \\
\text { finding } \\
\text { singular } \\
\text { strate- } \\
\text { gies }\end{array}$ & Calculus of variations & $\begin{array}{l}\text { Theory of optimal con- } \\
\text { trol }\end{array}$ \\
\hline $\begin{array}{l}\text { Selection } \\
\text { gradient }\end{array}$ & $\begin{array}{l}F_{s}\left(a, s(a), s^{\prime}(a)\right) \\
\mathrm{d} a\end{array} F_{s^{\prime}}\left(a, s(a), s^{\prime}(a)\right)$ & \\
\hline \begin{tabular}{l} 
for $a_{\min }<a<a_{\max }$ \\
\hline
\end{tabular} & - \\
\hline
\end{tabular}

\section{The seasonal flowering model by Dieckmann et al. (2006)}

Before presenting examples of applying the methods outlined above, we summarize the model upon which the examples are built. Following Dieckmann et al. (2006), we study the evolution of seasonal flowering schedules in plants that inhabit a temporally varying environment. Flowers opening at different times of the season experience different ecological conditions. Let $s(a)$ describe the flowering intensity of a plant at time $a$, with $a$ scaled so that $0 \leqslant a \leqslant 1$. The flowering intensity is naturally represented as a functionvalued trait (Dieckmann et al., 2006; Parvinen et al., 2006). Evidently, all flowering intensities are non-negative, $s(a) \geqslant 0$. Flowers produced at any time during the flowering season compete for pollinators and herbivore-free space. The effect of such competition is to decrease a plant's probability

$$
p\left(\frac{N s(a)}{K(a)}\right)
$$

of setting seed, where $N$ is the plant's population size, and $N s(a)$ is thus the total number of flowers open at time $a$. The time-dependent carrying capacity $K(a)$ describes seasonal factors affecting pollination success. The function $p$ is assumed to be decreasing from a maximum of $1: p(0)=1$ and $p^{\prime}(x) \leqslant 0$ for all $x \geqslant 0$. In the specific examples below, we use the functions

$$
p(x)=e^{-x}
$$


and

$$
K(a)=100\left(2+\sin \left(2 \pi\left((a-1)^{2}-1 / 4\right)\right)\right) .
$$

Plants having a larger flowering intensity than other plants attract more pollinators. We assume that this asymmetric competition affects the probability of setting seed according to a factor $g(\delta)$, where $\delta=\left(s_{\text {mut }}-s_{\text {res }}\right) / s_{\text {res }}^{\beta}$ describes the difference of flowering intensities, measured in a way that ranges from absolute $(\beta=0)$ to relative $(\beta=1)$. The function $g$ is assumed to have the properties $g(0)=1$ and $g^{\prime}(0)=\alpha / 2>0$. In the specific examples below, we use the function

$$
g(\delta)=\frac{2}{1+e^{-\alpha \delta}} .
$$

The total production of seeds can now be calculated by integrating reproductive success over the flowering season. Therefore, fitness is given by an integral of form (1), where the environment $E_{\text {res }}$ is determined by $s_{\text {res }}$ and $N_{\text {res }}$,

$$
\begin{aligned}
& r\left(s_{\mathrm{mut}}, s_{\mathrm{res}}, N_{\mathrm{res}}\right) \\
& =\int_{0}^{1} s_{\mathrm{mut}}(a)\left[p\left(\frac{s_{\mathrm{res}}(a) N_{\mathrm{res}}}{K(a)}\right) g\left(\frac{s_{\mathrm{mut}}(a)-s_{\mathrm{res}}(a)}{s_{\text {res }}(a)^{\beta}}\right)\right] \mathrm{d} a-d,
\end{aligned}
$$

where $d$ is the death rate of plants. The resident population size $N_{\text {res }}$ can be solved from the condition for ecological equilibrium,

$$
r\left(s_{\text {res }}, s_{\text {res }}, N_{\text {res }}\right)=0 .
$$

The production of flowers according to a schedule $s(a)$ requires resources. Dieckmann et al. (2006) assumed that flowering schedules are for this reason constrained by the inequality constraint

$$
\int_{0}^{1} s(a) \mathrm{d} a \leqslant 1
$$

Dieckmann et al. (2006) used this model as an illustration of the use of the canonical equation of function-valued traits, and in this manner were the first to demonstrate that evolutionary branching can occur also in functionvalued traits. As they already noted, the model summarized above is rather simplistic: this fact was reflected by the observation of evolutionary branching resulting in two coexisting strategies with completely separate flowering seasons, which implies abrupt changes in their flowering schedules (Dieckmann et al., 2006, their Fig. 3). Actually, such unrealistically complete temporal separation could become even more extreme, since the model allows evolutionary branching to occur over and over again, until infinitely 
many strategies coexist whose flowering periods are infinitesimally short and non-overlapping. In nature, such abrupt changes are unrealistic. For this reason, we study in this article three extensions of the seasonal flowering model of Dieckmann et al. (2006); these extensions are intended to add more ecological realism to the model, and to serve as examples of the use of theory presented in this article.

In the first extension (section 4), we add a cost of plasticity directly to the fitness function (21). Fitness is thus expressed as an integral that depends on the strategy $s(a)$ and its derivative $s^{\prime}(a)$, which means that it remains a direct-effect model of form (1). Parvinen et al. (2006) already showed how to use the calculus of variations to find singular strategies in models of this type. Here we illustrate how to use the canonical equation with derivative dependence (section 2.1) to study transient adaptive dynamics and evolutionary convergence to the previously identified singular strategy.

The second (section 5) and third (section 6) extensions result in processmediated models of form (2), for which the calculus of variations is no longer applicable. In the resultant more mechanistic setups, the strategy $s(a)$ describes how resources are used, and the actual flowering schedule $y(a)$ is obtained from a (system of) ordinary differential equation(s) affected by $\mathrm{s}(\mathrm{a})$. These extensions are used to show how the theory of optimal control (section 2.2) can help to find singular strategies. For better comparison of methodologies, we also calculate the selection gradient for both examples, even though this is rather tedious. While it is not feasible to solve the equilibria of the canonical equation (3) analytically, the canonical-equation dynamics can be calculated numerically.

\section{First extension: adding a direct cost of plas- ticity using the derivative}

In general, mechanisms that accurately separate the flowering intensities between two nearby moments in time can be expected to be costly. Therefore, in the first model extension, we assume that deviations of the derivative $s_{\text {mut }}^{\prime}(a)=\frac{\mathrm{d}}{\mathrm{d} a} s_{\text {mut }}(a)$ from zero have a direct negative effect on fitness,

$$
\begin{aligned}
r\left(s_{\text {mut }}, s_{\text {res }}, N_{\text {res }}\right)= & \int_{0}^{1}\left[s_{\text {mut }}(a) p\left(\frac{s_{\text {res }}(a) N_{\text {res }}}{K(a)}\right) g\left(\frac{s_{\text {mut }}(a)-s_{\text {res }}(a)}{s_{\text {res }}(a)^{\beta}}\right)\right. \\
& \left.-\kappa\left(s_{\text {mut }}^{\prime}(a)\right)^{2}\right] \mathrm{d} a-d,
\end{aligned}
$$

where $\kappa$ measures the strength of these plasticity costs. 


\subsection{Selection gradient}

By differentiating the integrand in equation (24), we obtain the derivatives

$$
\begin{aligned}
F_{s_{\mathrm{mut}}}( & \left(a, s_{\mathrm{mut}}(a), s_{\mathrm{res}}(a)\right)=\left[g\left(\frac{s_{\mathrm{mut}}(a)-s_{\mathrm{res}}(a)}{s_{\mathrm{res}}(a)^{\beta}}\right)\right. \\
+ & \left.s_{\mathrm{mut}}(a) g^{\prime}\left(\frac{s_{\mathrm{mut}}(a)-s_{\mathrm{res}}(a)}{s_{\mathrm{res}}(a)^{\beta}}\right) \frac{1}{s_{\mathrm{res}}(a)^{\beta}}\right] p\left(\frac{N s_{\mathrm{res}}(a)}{K(a)}\right)
\end{aligned}
$$

and $F_{s_{\text {mut }}^{\prime}}\left(s_{\text {mut }}^{\prime}(a)\right)=-2 \kappa s_{\text {mut }}^{\prime}(a)$, and thus the selection gradient (6),

$$
\begin{aligned}
G(a) & =F_{s_{\mathrm{mut}}}\left(a, s_{\mathrm{mut}}(a), s_{\mathrm{res}}(a)\right)-\left.\frac{\mathrm{d}}{\mathrm{d} a} F_{s_{\mathrm{mut}}^{\prime}}\left(s_{\mathrm{mut}}^{\prime}(a)\right)\right|_{s_{\mathrm{mut}}=s_{\mathrm{res}}=s} \\
& =\left(1+\frac{\alpha}{2} s(a)^{1-\beta}\right) p\left(\frac{N s(a)}{K(a)}\right)+2 \kappa s^{\prime \prime}(a)
\end{aligned}
$$

\subsection{Singular strategies with calculus of variations}

Because of the inequality constraints $(23)$ and $s(a) \geqslant 0$, the Euler-Lagrange equation for singular strategies obtained with the calculus of variations is $G(a)=C>0$ for $s(a)>0$ and $G(a)<C$ for $s(a)=0$.

Note that the population size $N$ in (26) depends on the strategy $s$. Therefore, $N$ and $s(a)$ must be solved together from (22) and (26). For $\kappa=0$, this solution is found algebraically, but for $\kappa>0$, it requires solving a secondorder differential equation. The necessary boundary conditions, resulting from equation (21) of Parvinen et al. (2006) (see also Proposition 1), are $F_{s^{\prime}}=-2 \kappa s^{\prime}=0$ at either boundary, yielding $s^{\prime}(0)=0$ and $s^{\prime}(1)=0$.

(a)

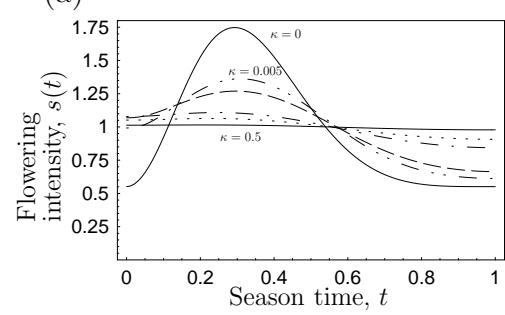

(b)

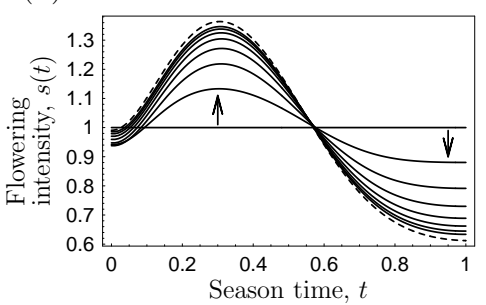

Figure 1: (a) Evolutionarily singular flowering strategies for different costs of plasticity, $\kappa=0,0.005,0.01,0.05,0.1,0.5$. (b) Evolution of the flowering strategy according to the canonical equation, from the initial strategy $s(a)=$ 1 towards the singular strategy, for $\kappa=0.005$. Other parameters: $\alpha=1$, $\beta=0.9$, and $d=0.5$. 
Figure 1a illustrates singular strategies for different values of the plasticity cost $\kappa$. Other parameters are chosen as in Dieckmann et al. (2006); therefore, the singular strategy for $\kappa=0$ in figure 1a matches that in figure 3 of Dieckmann et al. (2006). For $\kappa=0$, it is easy to show that any singular strategy can be invaded by some mutants with extremely concentrated flowering schedules, so such a singular strategy is not evolutionarily stable. For $\kappa>0$, the corresponding analysis is not straightforward, but our numerical results suggest that when $\kappa$ is large enough, the singular strategy turns into a fitness maximum, and thus becomes evolutionarily stable.

\subsection{Canonical-equation dynamics}

We have already calculated the selection gradient (26) for the model (24). It is needed for applying the canonical equation (3), which describes the rate of expected evolutionary change in a function-valued strategy (Dieckmann et al., 2006). The equilibrium population size $N$ for strategy $s$ is obtained from (24) by requiring $r(s, s, N)=0$. In addition, we need to specify the mutation probability $\mu(s)$ and the variance-covariance function $\sigma^{2}$ of the mutation distribution. Here we have assumed that $\sigma^{2}\left(a^{\prime}, a\right)=\sigma^{2}\left[\delta\left(a-a^{\prime}\right)-1\right]$, which means that an increase or decrease of $s$ at a particular argument value $a$ is compensated uniformly across all other argument values, thus ensuring that the trait normalization (23) stays intact. Note, however, that the choice of the variance-covariance function can substantially affect the dynamics and evolutionary equilibrium of the canonical equation (Heino et al., 2008, their figure 5).

Figure 1b illustrates the dynamics of the canonical equation starting from a uniform initial strategy $s(a)=1$. As expected, the monomorphic strategy approaches the singular strategy predicted by the calculus of variations (figure 1a).

\section{Second extension: process-mediated flower production through gradual resource allo- cation}

In the previous section, we have assumed that the flowering-intensity schedule at time $a$ within the season is the evolving strategy. However, it is more reasonable to assume that the flowering schedule is actually a result of the time-dependent intensity $s(t) \geqslant 0$ according to which resources are allocated to flower production, and that it is this allocation intensity that 
is the function-valued trait under natural selection. The flowering-intensity schedule thus becomes a state variable, denoted by $y(t)$, which is assumed to satisfy the differential equation

$$
\frac{\mathrm{d}}{\mathrm{d} t} y(t)=h(s(t))-\xi y(t), \quad y(0)=0,
$$

where the function $h(s)$ is the functional response of flower production, and $\xi$ is the rate at which flowers die. Since available resources are necessarily limited, we have the constraint

$$
\int_{0}^{1} s(t) \mathrm{d} t \leqslant S_{\max }
$$

but note that in practice plants should not leave available resources unused, so this inequality constraint will be satisfied as an equality constraint instead. Flower and seed dynamics are otherwise as in section 3, except that in this second example the cost of plasticity is included in the function $h$, so it is not necessary to include it in the fitness function as a dependence on $y^{\prime}(t)$. Furthermore, an inequality constraint on the total amount of flowers, as in (23), is not needed, because such a constraint follows from (28). We thus assume that fitness is given by

$$
r\left(s_{\mathrm{mut}}, E_{\mathrm{res}}\right)=\int_{0}^{1} \underbrace{y_{\mathrm{mut}}(a) p\left(\frac{y_{\mathrm{res}}(a) N_{\mathrm{res}}}{K(a)}\right) g\left(\frac{y_{\mathrm{mut}}(a)-y_{\mathrm{res}}(a)}{y_{\mathrm{res}}(a)^{\beta}}\right)}_{F\left(a, y_{\mathrm{mut}}(a), y_{\mathrm{res}}(a)\right)} \mathrm{d} a-d,
$$

where the environment $E_{\text {res }}$ created by the resident is characterized by the resident population size $N_{\text {res }}$ and the flowering intensity $y_{\text {res }}$ which solves the differential equation (27) for the resident allocation strategy $s_{\text {res }}$. Analogously, the flowering intensity $y_{\text {mut }}$ of the mutant solves the differential equation (27) for the mutant allocation strategy $s_{\text {mut }}$.

\subsection{Selection gradient}

Since the fitness function (29) is not of form (1), equation (6) for the selection gradient cannot be used. We thus need to employ equation (4) directly. Let us first note that the solution of the differential equation (27) with $s^{*}(t)=$ $s(t)+\epsilon \Delta s(t)$ can be written as

$$
y^{*}(t)=e^{-\xi t} \int_{0}^{t} e^{\xi \tau} h\left(s^{*}(\tau)\right) \mathrm{d} \tau=e^{-\xi t} \int_{0}^{t} e^{\xi \tau} h(s(\tau)+\epsilon \Delta s(\tau)) \mathrm{d} \tau .
$$


Thus we obtain

$$
\begin{aligned}
\left.\frac{\mathrm{d}}{\mathrm{d} \epsilon} y^{*}(t)\right|_{\epsilon=0} & =e^{-\xi t} \int_{0}^{t} e^{\xi \tau} h^{\prime}(s(\tau)) \Delta s(\tau) \mathrm{d} \tau \\
& \rightarrow \begin{cases}0, & \text { if } t<\hat{t} \\
e^{\xi(\hat{t}-t)} h^{\prime}(s(\hat{t})), & \text { if } t>\hat{t}\end{cases}
\end{aligned}
$$

when $\Delta s$ tends to the Dirac delta function $\delta_{\hat{t}}$. This result can be used in the calculation of the selection gradient,

$$
\begin{aligned}
G(a) & =\lim _{\Delta s \rightarrow \delta_{a}}\left[\left.\frac{\mathrm{d}}{\mathrm{d} \epsilon} r\left(s+\epsilon \Delta s, E_{\mathrm{res}}\right)\right|_{\epsilon=0}\right] \\
& =\left.\lim _{\Delta s \rightarrow \delta_{a}} \int_{0}^{1} F_{y_{\mathrm{mut}}}\left(t, y_{\mathrm{mut}}(t), y_{\mathrm{res}}(t)\right) \frac{\mathrm{d}}{\mathrm{d} \epsilon} y^{*}(t)\right|_{\epsilon=0} \mathrm{~d} t \\
& =h^{\prime}(s(a)) \int_{a}^{1} F_{y_{\mathrm{mut}}}\left(t, y_{\mathrm{mut}}(t), y_{\mathrm{res}}(t)\right) e^{\xi(a-t)} \mathrm{d} t
\end{aligned}
$$

Without constraints set on the evolving strategy $s$, singular strategies are strategies for which the selection gradient vanishes, $G(a)=0$. In the present model, increased allocation intensity will always increase flowering intensity, and thus fitness. Therefore, $G(a)>0$ for all $a<1$. Since allocatable resources are necessarily limited, we have the global inequality constraint (28), which for singular strategies is fulfilled with an equality. In addition, we have the constraint $s(a) \geqslant 0$, because allocation intensity is necessarily nonnegative. For a singular strategy, a fitness increase due to increased resource usage at one time must be exactly compensated by a fitness decrease due to decreased resource usage at other times. More precisely, for singular strategies $s$, a mutant strategy $s+\epsilon \Delta s$ belongs to the strategy space $S$ for small $|\epsilon|$ only if $\int_{a_{\min }}^{a_{\max }} \Delta s(t) \mathrm{d} t=0$, and if $\Delta s(a)=0$ for such $a$ for which $s(a)=0$. The definition (5) of singular strategies can only be fulfilled if the first-order fitness increase for increased resource usage at time $a$, which is measured by the selection gradient $G(a)$, is the same $(=C)$ for such $a$ for which $s(a)>0$. For such $a$ for which $s(a)=0$, the fitness increase must be less than $C$. Altogether, we obtain

$$
G(a)-C \begin{cases}=0, & \text { if } s(a)>0 \\ <0, & \text { if } s(a)=0\end{cases}
$$

where the constant $C>0$ is determined such that the constraint (28) holds with equality. See also section 2.3.2 of Parvinen et al. (2006). 


\subsection{Singular strategies with theory of optimal control}

The model (27-29) can be written in a standard format used in the theory of optimal control (11),

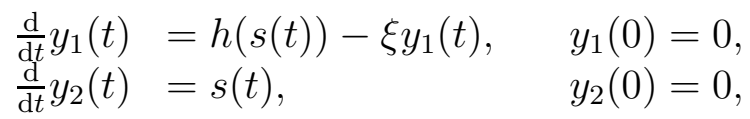

with the condition $y_{2}(1) \leqslant S_{\max }$, so that $(28)$ is satisfied. As explained before, this condition will actually be satisfied with equality, $y_{2}(1)=S_{\max }$. Now the Hamiltonian (13) is given by

$$
H=F\left(t, y_{1}(t)\right)+\lambda_{1}\left(h(s(t))-\xi y_{1}(t)\right)+\lambda_{2}(t) s(t) .
$$

Because the Hamiltonian does not depend on $y_{2}$, the condition (12) for $\lambda_{2}$ is

$$
\frac{\mathrm{d}}{\mathrm{d} t} \lambda_{2}(t)=-H_{y_{2}}=0,
$$

so $\lambda_{2}(t)$ does not depend on $t, \lambda_{2}(t)=\lambda_{2}$. Note that since the value of $y_{2}(1)$ is fixed, the value of $\lambda_{2}(1)$ is free, and is set to satisfy $y_{2}(1)=S_{\max }$. For $\lambda_{1}$, we obtain from (12) the differential equation

$$
\frac{\mathrm{d}}{\mathrm{d} t} \lambda_{1}(t)=-H_{y_{1}(t)}=-F_{y_{1}(t)}+\lambda_{1}(t) \xi \quad \text { with } \lambda_{1}(1)=0 .
$$

Because $F_{y_{1}}>0$, the solution of (37) satisfies $\lambda_{1}(t) \geqslant 0$ for $0 \leqslant t \leqslant 1$. In each moment, $s(t)$ is chosen such that the Hamiltonian is maximized. The only terms in the Hamiltonian (35) that depend on $s$ are $\lambda_{1} h(s(t))$ and $\lambda_{2} s(t)$. If $\lambda_{2} \geqslant 0$, the Hamiltonian is maximized by $s(t) \rightarrow \infty$, but that solution cannot satisfy the constraint $y_{2}(1) \leqslant S_{\max }$. Therefore, $\lambda_{2}<0$, and possible values for $s(t)$ are

$$
\begin{aligned}
& s(t)=0, \quad \text { if } \lambda_{1}(t) h^{\prime}(0)+\lambda_{2}<0 \\
& s(t)>0, \quad \text { such that } \lambda_{1}(t) h^{\prime}(s(t))+\lambda_{2}=0 .
\end{aligned}
$$

Especially, if the functional response $h$ is strictly concave, $\left(h^{\prime \prime}(s)<0\right)$, equation (38b) has no solution if (38a) holds, and a unique solution if (38a) does not hold.

We thus obtain the singular strategy $s(t)$ by solving the differential equations (34) and (37) together with (38) from $t=0$ with two unknowns: $\lambda_{1}(0)$ and $\lambda_{2}$, which are used to satisfy the conditions $y_{2}(1)=S_{\max }$ and $\lambda_{1}(1)=0$. In addition, we have the condition $r(s, N)=0$ for a population at ecological equilibrium, and thus this is a problem of optimal control with environmental 
feedback. Another option is to start solving the differential equations from $t=1$ with initial conditions $\lambda_{1}(1)=0$ and $y_{2}(1)=S_{\max }$, and then to use the unknowns $\lambda_{2}$ and $y_{1}(1)$ to satisfy the conditions $y_{1}(0)=y_{2}(0)=0$.

In our numerical explorations we obtained better accuracy using the second alternative. For the numerical examples below, we use the same parameters as Dieckmann et al. (2006) and assume that the functional response of flower production is of Holling type II,

$$
h(s)=\frac{s}{1+\gamma s} .
$$

Therefore, the possible values for $s(t)$ from condition (38) are

$$
\begin{cases}s(t)=0, & \text { if } 0 \leqslant \lambda_{1}(t) \leqslant-\lambda_{2} \\ s(t)=\frac{1}{\gamma}\left(\sqrt{-\frac{\lambda_{1}(t)}{\lambda_{2}}}-1\right) & \text { if } \lambda_{1}(t)>-\lambda_{2}>0 .\end{cases}
$$

Figure 2a illustrates singular allocation intensities $s(t)$ obtained with the optimal control method described above for different values of $\gamma$. Figure $2 \mathrm{~b}$ illustrates the resulting flowering schedules. For comparison, Figure 2c illustrates the selection gradient calculated from (32) for the singular strategy obtained with optimal control theory; this shows that the condition (33) is satisfied. Note, however, that the theory of optimal control only gives necessary conditions for a strategy to be a fitness maximum, and thus uninvadable. Unlike in the calculus of variations, there is no general theory that would provide sufficient conditions for fitness maxima. Furthermore, the theory of optimal control cannot be used to study whether a singular strategy is convergence stable or not. For this purpose, we need to investigate the corresponding canonical-equation dynamics, which we therefore do next.

\subsection{Canonical-equation dynamics}

Figure 3 illustrates the canonical-equation dynamics (3) of the model (2729) starting from a uniform allocation schedule, $s(a)=2$. As in our first example, we again assume that $\sigma^{2}\left(a^{\prime}, a\right)=\sigma^{2}\left[\delta\left(a-a^{\prime}\right)-1\right]$. We observe that the allocation schedule converges to the same singular strategy that was obtained with the theory of optimal control, as illustrated in figure 2. Our numerical investigations suggest that this singular strategy is uninvadable, and thus an evolutionary endpoint.

Figure 4a-c illustrates monomorphic canonical-equation dynamics for another set of parameter values. In this case, however, the monomorphic singular strategy is not uninvadable. In such a case, evolutionary branching 
(a)

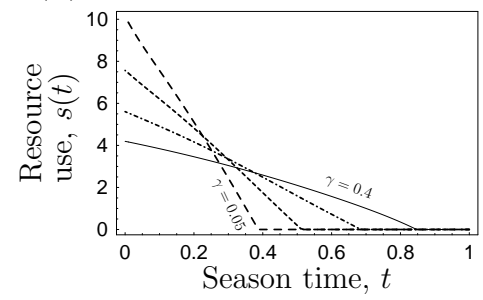

(b)

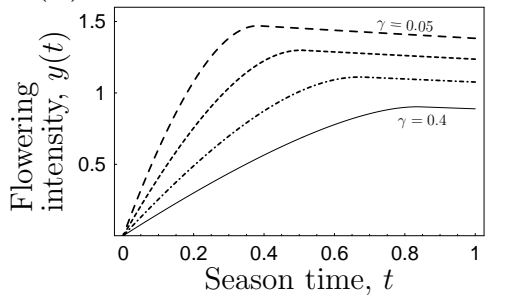

(c)

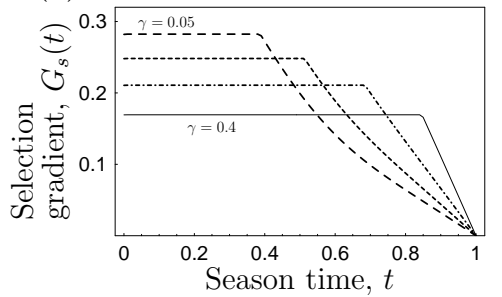

Figure 2: Evolutionarily singular strategies in the model (27-29) obtained with the theory of optimal control for different functional-response parameters, $\gamma=0.05,0.1,0.2$, and 0.4. (a) Allocation schedule $s(t)$. (b) Resultant flowering schedule $y(t)$. (c) Selection gradient according to equation (32) for $\gamma=0.1$. Other parameters: $\alpha=1, \beta=0.9, \xi=0.1, S_{\max }=2$, and $d=0.5$.

can occur. Figure 4d-e illustrates dimorphic canonical-dynamics towards an evolutionarily singular coalition of two strategies, shown for greater clarity in Figure 4f-g, with the corresponding two selection gradients illustrated in Figure $4 \mathrm{~h}$. While the two resource allocation schedules are strictly segregated in time, the resulting flowering schedules show considerable overlap. Furthermore, the dimorphic flowering schedules we obtain for this extended model are much smoother than those in the original model of Dieckmann et al. (2006).

\section{Third extension: process-mediated flower production through gradual and differen- tial resource allocation}

As another example of a process-mediated model formulation, we study the third extension of the flowering model by Dieckmann et al. (2006). As in the second extension, the function-valued trait affects a process of flower growth, but this time the trait is about resource allocation between leaf growth and flower production.

Let $x(t)$ denote the density of leafs in a plant and $y(t)$ the density of flowers (flowering intensity). The leaf density at the beginning of season is $x_{0}$. Assume that there is incoming energy (from the sunlight) with intensity $E(t)$, and the plant uses its leafs to collect that energy. The plant allocates the proportion $s(t) \geqslant 0$ to flower production and $1-s(t) \geqslant 0$ to leaf growth. There is a functional response in both duties described by the functions $f$ 
(a)

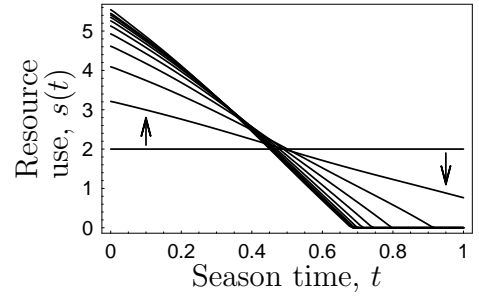

(b)

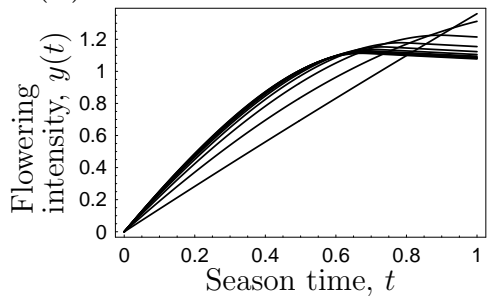

(c)

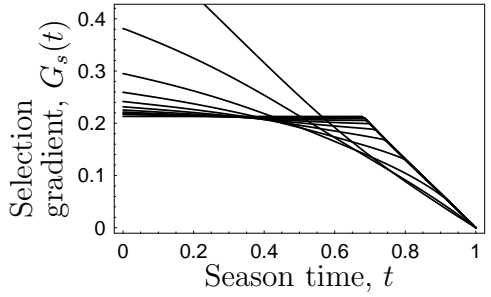

Figure 3: Canonical-equation dynamics of the model (27-29), starting from a uniform allocation schedule, $s(a)=2$. (a) Allocation schedule $s(t)$. (b) Resultant flowering schedule $y(t)$. (c) Selection gradient according to equation (32). The strategy converges towards the same singular strategy as shown in Figure 2. Other parameters: $\alpha=1, \beta=0.9, \gamma=0.2, \xi=0.1$, $S_{\max }=2$, and $d=0.5$.

and $h$, respectively. The nonlinearity described by the function $h$ occurs at the (global) plant level, while the nonlinearity described by the function $f$ occurs at the (local) level of leave tissue. The latter assumption is made also because of technical reasons. Furthermore, leafs decay with the rate $\omega$ and flowers with the rate $\xi$. As a result, we obtain the system of differential equations

$$
\begin{aligned}
& \frac{\mathrm{d} x(t)}{\mathrm{d} a}=x(t) f(E(t)(1-s(t)))-\omega x(t) \quad x(0)=x_{0} \\
& \frac{\mathrm{d} y(t)}{\mathrm{d} t}=h(E(t) s(t) x(t))-\xi y(t) \quad y(0)=0 .
\end{aligned}
$$

Otherwise the model is as described before, and thus fitness is given by the equation (29), which is of the process-mediated form (10).

\subsection{Selection gradient}

As in the second extension, the fitness function is not of form (1). Therefore the formula (6) for the selection gradient cannot be used and equation (4) needs to be used directly. For this purpose note that the differential equation for the leaf density $x(t, s)$ in the system of differential equations (41) is linear with respect to $x(t, s)$ and does not depend on $y(t, s)$. Therefore the solution is

$$
x(t, s)=x_{0} \exp \left(\int_{0}^{t} f(E(\tau)(1-s(\tau)))-\omega \mathrm{d} \tau\right) .
$$


Using the notation $Q(t, s)=h(E(t) s(t) x(t))$, we can write the solution for flowering intensity $y(t, s)$ as

$$
y(t, s)=e^{-\xi t} \int_{0}^{t} Q(\tau, s) e^{\xi \tau} \mathrm{d} \tau
$$

From equation (42) we obtain

$$
\begin{aligned}
x^{*}(t, s, \Delta s) & =\left.\frac{\mathrm{d}}{\mathrm{d} \epsilon} x(t, s+\epsilon \Delta s)\right|_{\epsilon=0} \\
& =x(t, s) \int_{0}^{t} f^{\prime}(E(\tau)(1-s(\tau)))(-E(\tau) \Delta s(\tau)) \mathrm{d} \tau
\end{aligned}
$$

and the limit when $\Delta s(\tau)$ tends to the Dirac delta function $\delta_{\hat{t}}$ is

$$
\lim _{\Delta s \rightarrow \delta_{\hat{t}}} x^{*}(t, s, \Delta s)= \begin{cases}0 & \text { when } t<\hat{t}, \\ -x(t, s) E(\hat{t}) f^{\prime}(E(\hat{t})(1-s(\hat{t}))) & \text { when } t>\hat{t} .\end{cases}
$$

From (43) we obtain the derivative

$$
\begin{aligned}
& y^{*}(t, s, \Delta s)=\left.\frac{\mathrm{d}}{\mathrm{d} \epsilon} y(t, s+\epsilon \Delta s)\right|_{\epsilon=0}= \\
& e^{-\xi t} \int_{0}^{t} h^{\prime}(E(\tau) s(\tau) x(\tau, s)) E(\tau)\left(\Delta s(\tau) x(\tau, s)+s(\tau) x^{*}(\tau, s, \Delta s)\right) e^{\xi \tau} \mathrm{d} \tau
\end{aligned}
$$

We also need the limit for $y^{*}(t, s, \Delta s)$, when $\Delta s(\tau)$ tends to the Dirac delta function $\delta_{\hat{t}}$. Since changing the resource use with $\epsilon \delta_{\hat{t}}$ can only affect flowering intensities at time $\hat{t}$ and after, we have $\lim _{\Delta s \rightarrow \delta_{\hat{t}}} y^{*}(t, s, \Delta s)=0$ when $t<\hat{t}$. For $t \geqslant \hat{t}$ we have

$$
\begin{gathered}
\hat{y}(t, s, \hat{t})=\lim _{\Delta s \rightarrow \delta_{\hat{t}}} y^{*}(t, s, \Delta s)=e^{-\xi t} E(\hat{t})\left[h^{\prime}(E(\hat{t}) s(\hat{t}) x(\hat{t}, s)) x(\hat{t}, s) e^{\xi \hat{t}}\right. \\
\left.-f^{\prime}(E(\hat{t})(1-s(\hat{t}))) \int_{\hat{t}}^{t} h^{\prime}(E(\tau) s(\tau) x(\tau, s)) E(\tau) s(\tau) x(\tau, s) e^{\xi \tau} \mathrm{d} \tau\right] .
\end{gathered}
$$

Finally, the selection gradient is

$$
G(a)=\lim _{\Delta s \rightarrow \delta_{a}}\left[\left.\frac{\mathrm{d}}{\mathrm{d} \epsilon} r(s+\epsilon \Delta s)\right|_{\epsilon=0}\right]=\int_{a}^{1} F_{y_{\mathrm{mut}}}(t) \hat{y}(t, s, a) \mathrm{d} t .
$$

The selection gradient is thus obtained with double integration, which is tedious, but can be done numerically for any known strategy. Therefore calculating the canonical-equation dynamics and waiting until it converges can be used to find singular strategies numerically. However, to solve singular strategies directly from equation (48) is out of our scope. Instead, it is relatively easy to use the theory of optimal control for that purpose. 


\subsection{Singular strategies with theory of optimal control}

Like the second extension, the third model formulation (41) can be written in a standard format of theory of optimal control (11) in the following way:

$$
\begin{aligned}
& \frac{\mathrm{d} y_{1}(t)}{\mathrm{d} t}=y_{1}(t) f(E(t)(1-s(t)))-\omega y_{1}(t), \quad y_{1}(0)=x_{0}, \\
& \frac{\mathrm{d} y_{2}(t)}{\mathrm{d} t}=h\left(E(t) s(t) y_{1}(t)\right)-\xi y_{2}(t), \quad y_{2}(0)=0 .
\end{aligned}
$$

Now the Hamiltonian (13) is

$$
\begin{aligned}
H= & F\left(t, y_{2}\right)+\lambda_{1}(t) \frac{\mathrm{d} y_{1}(t)}{\mathrm{d} t}+\lambda_{2}(t) \frac{\mathrm{d} y_{2}(t)}{\mathrm{d} t} \\
= & y_{2}(t) p^{*}(t) g^{*}\left(y_{2}(t), t\right)+\lambda_{1}(t)\left[y_{1}(t) f(E(t)(1-s(t)))-\omega y_{1}(t)\right] \\
& +\lambda_{2}(t)\left[h\left(E(t) s(t) y_{1}(t)\right)-\xi y_{2}(t)\right],
\end{aligned}
$$

where $p^{*}(t)=p\left(\frac{y_{\mathrm{res}}(t) N_{\mathrm{res}}}{K(t)}\right)$ and $g^{*}\left(y_{2}(t), t\right)=g\left(\frac{y_{2}(t)-y_{\mathrm{res}}(t)}{y_{\mathrm{res}}(t)^{\beta}}\right)$. The conditions (12) for $\lambda_{1}$ and $\lambda_{2}$ are

$$
\begin{aligned}
\frac{\mathrm{d}}{\mathrm{d} t} \lambda_{1}(t)=-H_{y_{1}}= & -\lambda_{1}(t)[f(E(t)(1-s(t)))-\omega] \\
& -\lambda_{2}(t) h^{\prime}\left(E(t) s(t) y_{1}(t)\right) E(t) s(t)
\end{aligned}
$$

and

$$
\frac{\mathrm{d}}{\mathrm{d} t} \lambda_{2}(t)=-H_{y_{2}}=-p^{*}(t)\left[g^{*}\left(y_{2}(t), t\right)+y_{2}(t) \frac{\partial}{\partial y_{2}(t)} g^{*}\left(y_{2}(t), t\right)\right]+\lambda_{2}(t) \xi
$$

with $\lambda_{1}(1)=\lambda_{2}(1)=0$.

We thus obtain the singular strategy $s(t)$ by solving the differential equations (49), (51) and (52) and choosing $s(t)$ such that it maximizes the Hamiltonian (50) at each point. In addition we have the population size condition $r(s, N)=0$, and thus this is a problem of optimal control with environmental feedback. We can either start from $t=0$ with two unknowns: $\lambda_{1}(0)$ and $\lambda_{2}(0)$, which are used to get conditions $\lambda_{1}(1)=\lambda_{2}(1)=0$ satisfied. Another option is to start solving the differential equations from $t=1$ with initial conditions $\lambda_{1}(1)=\lambda_{2}(1)=0$, and use the unknowns $y_{1}(1)$ and $y_{2}(1)$ to get the conditions $y_{1}(0)=x_{0}$ and $y_{2}(0)=0$ satisfied.

Figure 5a illustrates singular resource allocation strategies $s(t)$ obtained with the optimal control method described above for different values of $x_{0}$. Figure 5b illustrates the resulting leaf intensity and Figure $5 \mathrm{c}$ the flowering schedules. For comparison purposes, Figure 5d illustrates the selection gradients calculated from (48) with the singular strategy obtained with optimal control theory. 


\subsection{Canonical-equation dynamics}

Figure 6 illustrates the canonical-equation dynamics (3) in the model (41) starting from a uniform resource use strategy $s(a)=0.75$. This time we have assumed that $\sigma^{2}\left(a^{\prime}, a\right)=\sigma^{2} \delta\left(a-a^{\prime}\right)$, which means that increase or decrease of $s$ at a particular argument value has no effect on other argument values. We observe that the strategy of the evolving population approaches the same singular strategy that was obtained with the theory of optimal control and illustrated in figure 5 for $x_{0}=0.05$.

\section{Conclusions}

In this article we have further developed the theory of adaptive dynamics of function-valued traits. Previously, we had shown how the calculus of variations can be used to find singular strategies in direct-effect models, in which the invasion fitness can be written as an integral, in which the integrand for each argument value is a function of the strategy value at that point only (Parvinen et al., 2006). Here we have broadened our methods of analysis to tackle process-mediated models, a rather general class of models that includes direct-effect models as a subset. In particular, we have described how to use optimal control theory to find singular strategies in process-mediated models, in which the function-valued strategy affects a process described by differential equations, and fitness can be expressed as an integral, with the integrand depending both on the strategy and process variables. Our results show that singular strategies of such models need to satisfy Pontryagin's maximum principle with environmental feedback.

Although the class of process-mediated models is wide, not all models of interest are of such form. For example, the metapopulation model in Section 7 of Parvinen et al. (2006) is an example of a direct-effect model in which invasion fitness is a function of several integrals. Although Euler's equation of the calculus of variations cannot be used directly to analyze that model, Parvinen et al. (2006) have shown how to adapt the derivation of Euler's equation to find singular strategies in that model. This approach is applicable also for solving other direct-effect models in which invasion fitness is a function of several integrals. Analogously, it is straightforward to construct process-mediated models in which invasion fitness is a function of several integrals. For such models, Pontryagin's maximum principle cannot be used directly, but one may be able to adapt its derivation.

The expansion of the theory of adaptive dynamics of function-valued traits developed here is not only mathematically interesting, but also al- 
lows us to investigate biologically more realistic models. We hope this work will further stimulate the application of function-valued traits in theoretical evolutionary ecology. For example, here we have shown how function-valued traits can be used to study evolution of flowering schedules. Our model in Section 5 shows that in presence of asymmetric competition, temporal segregation in flowering schedules can evolve. Known as staggered flowering, this phenomenon is known in tropical plant communities and could be a mechanism for reproductive isolation between closely related species (Snow, 1965; Stiles, 1977). We plan to explore staggered flowering in a forthcoming paper.

\section{A Proof of proposition 1}

Proof. When the fitness function is of form (1), differentiation results in (See also Equation 10 of Parvinen et al. (2006))

$$
\begin{gathered}
\left.\frac{\mathrm{d}}{\mathrm{d} \epsilon} r(s+\epsilon \Delta s)\right|_{\epsilon=0}=\left.\int_{a_{\min }}^{a_{\max }} \frac{\mathrm{d}}{\mathrm{d} \epsilon} F\left(a, s(a)+\epsilon \Delta s, s^{\prime}(a)+\epsilon \Delta s^{\prime}\right) \mathrm{d} a\right|_{\epsilon=0} \\
\quad=\int_{a_{\min }}^{a_{\max }} F_{s}\left(a, s(a), s^{\prime}(a)\right) \Delta s+F_{s^{\prime}}\left(a, s(a), s^{\prime}(a)\right) \Delta s^{\prime} \mathrm{d} a .
\end{gathered}
$$

Integration by parts yields

$$
\begin{aligned}
& \left.\frac{\mathrm{d}}{\mathrm{d} \epsilon} r(s+\epsilon \Delta s)\right|_{\epsilon=0}=\int_{a_{\min }}^{a_{\max }}\left[F_{s}\left(a, s(a), s^{\prime}(a)\right)-\frac{\mathrm{d}}{\mathrm{d} a} F_{s^{\prime}}\left(a, s(a), s^{\prime}(a)\right)\right] \Delta s \mathrm{~d} a \\
& \quad+F_{s^{\prime}}\left(a_{\max }, s\left(a_{\max }\right), s^{\prime}\left(a_{\max }\right)\right) \Delta s\left(a_{\max }\right) \\
& \quad-F_{s^{\prime}}\left(a_{\min }, s\left(a_{\min }\right), s^{\prime}\left(a_{\min }\right)\right) \Delta s\left(a_{\min }\right) .
\end{aligned}
$$

For $\Delta s \rightarrow \delta_{a}$ with $a_{\min }<a<a_{\max }$, both $\Delta s\left(a_{\min }\right) \rightarrow 0$ and $\Delta s\left(a_{\max }\right) \rightarrow 0$, and the integral tends to the value of the integrand at $a$, which gives

$$
G(a)=F_{s}\left(a, s(a), s^{\prime}(a)\right)-\frac{\mathrm{d}}{\mathrm{d} a} F_{s^{\prime}}\left(a, s(a), s^{\prime}(a)\right) \quad \text { for } a_{\min }<a<a_{\max } .
$$

However, for $\Delta s \rightarrow \delta_{a}$ with $a=a_{\min }, \Delta s\left(a_{\text {min }}\right) \rightarrow \infty$; the corresponding limit for $a=a_{\text {max }}$ is also infinite. Therefore, unless $F_{s^{\prime}}=0$ at a boundary, the selection pressure at that boundary is an order of magnitude stronger than elsewhere, forcing $F_{s^{\prime}}$ to become zero practically immediately compared to the speed of evolution elsewhere, from which the result follows.

Acknowledgements: K.P. wishes to thank the Academy of Finland for financial support (grant number 128323). M.H. acknowledges support from 
the Bergen Research Foundation U.D. gratefully acknowledges financial support by the European Science Foundation, the Austrian Science Fund, the Austrian Ministry of Science and Research, and the Vienna Science and Technology Fund, as well as by the European Commission, through the Marie Curie Research Training Network FishACE and the Specific Targeted Research Project FinE.

\section{References}

Beder, J. H. and R. Gomulkiewicz (1998). Computing the selection gradient and evolutionary response of an infinite-dimensional trait. J. Math. Biol. 36, 299-319.

Calsina, Á. and J. Ripoll (2010). Evolution of age-dependent sex-reversal under adaptive dynamics. J. Math. Biol. 60(2), 161-188.

Christiansen, F. B. (1991). On conditions for evolutionary stability for a continuously varying character. Am. Nat. 138, 37-50.

Dieckmann, U., M. Heino, and K. Parvinen (2006). The adaptive dynamics of function-valued traits. J. Theor. Biol 241, 370-389.

Dieckmann, U. and R. Law (1996). The dynamical theory of coevolution: A derivation from stochastic ecological processes. J. Math. Biol. 34, 579-612.

Ernande, B., U. Dieckmann, and M. Heino (2004). Adaptive changes in harvested populations: plasticity and evolution of age and size at maturation. Proc. R. Soc. London B 271, 415-423.

Eskola, H. (2009). On the evolution of timing of reproduction. Theor. Popul. Biol. 75, 98-108.

Eskola, H. T. M., S. A. H. Geritz, and M. Gyllenberg (2011). On the evolution of the timing of reproduction with non-equilibrium resident dynamics. Bull. Math. Biol. 73(6), 1312-1332.

Geritz, S. A. H., É. Kisdi, G. Meszéna, and J. A. J. Metz (1998). Evolutionarily singular strategies and the adaptive growth and branching of the evolutionary tree. Evol. Ecol. 12, 35-57.

Geritz, S. A. H., J. A. J. Metz, É. Kisdi, and G. Meszéna (1997). Dynamics of adaptation and evolutionary branching. Phys. Rev. Lett. 78, 2024-2027. 
Gilchrist, M., D. Sulsky, and A. Pringle (2006). Identifying fitness and optimal life-history strategies for an asexual filamentous fungus. Evolution 60, 970-979.

Gomulkiewicz, R. and J. H. Beder (1996). The selection gradient of an infinite-dimensional trait. SIAM J. Appl. Math 56, 509-523.

Gomulkiewicz, R. and M. Kirkpatrick (1992). Quantitative genetics and the evolution of reaction norms. Evolution 46, 390-411.

Gyllenberg, M., É. Kisdi, and M. Utz (2008). Evolution of conditiondependent dispersal under kin competition. J. Math. Biol. 57(2), 285-307.

Hamelin, F. M. and M. A. Lewis (2010). A differential game theoretical analysis of mechanistic models for territoriality. J. Math. Biol. 61(5), 665-694.

Heino, M., K. Parvinen, and U. Dieckmann (2008). Evolution of foraging strategies on resource gradients. Evol. Ecol. Res. 10, 1131-1156.

Jaffrézic, F. and S. D. Pletcher (2000). Statistical models for estimating the genetic basis of repeated measures and other function-valued traits. Genetics 156, 913-922.

Kingsolver, J. G., R. Gomulkiewicz, and P. A. Carter (2001). Variation, selection and evolution of function-valued traits. Genetica 112-113, 87104.

Leimar, O. (2001). Evolutionary change and Darwinian demons. Selection 2, $65-72$

Marrow, P., U. Dieckmann, and R. Law (1996). Evolutionary dynamics of predator-prey systems: An ecological perspective. J. Math. Biol. 34, 556578.

Matessi, C. and C. Di Pasquale (1996). Long-term evolution of multilocus traits. J. Math. Biol. 34, 613-653.

Maynard Smith, J. (1976). Evolution and the theory of games. Amer. Sci. 64, $41-45$

Maynard Smith, J. (1982). Evolution and the Theory of Games. Cambridge University Press, Cambridge. 
Meszéna, G., É. Kisdi, U. Dieckmann, S. A. H. Geritz, and J. A. J. Metz (2001). Evolutionary optimisation models and matrix games in the unified perspective of adaptive dynamics. Selection 2, 193-210.

Metz, J. A. J., S. A. H. Geritz, G. Meszéna, F. J. A. Jacobs, and J. S. van Heerwaarden (1996). Adaptive dynamics, a geometrical study of the consequenses of nearly faithful reproduction. In S. J. van Strien and S. M. Verduyn Lunel (Eds.), Stochastic and Spatial Structures of Dynamical Systems, pp. 183-231. North-Holland, Amsterdam.

Metz, J. A. J., R. M. Nisbet, and S. A. H. Geritz (1992). How should we define "fitness" for general ecological scenarios? Trends Ecol. Evol. 7, $198-202$.

Parvinen, K., U. Dieckmann, and M. Heino (2006). Function-valued adaptive dynamics and the calculus of variations. J. Math. Biol. 52, 1-26.

Perrin, N. and R. M. Sibly (1993). Dynamic-models of energy allocation and investment. Annu. Rev. Ecol. Syst. 24, 379-410.

Pontryagin, L. S., V. G. Boltayanskii, R. V. Gamkrelidze, and E. F. Mishchenko (1962). The Mathematical Theory of Optimal Processes. Wiley, New York.

Rees, M. and S. P. Ellner (2009). Integral projection models for populations in temporally varying environments. Ecol. Monogr. $79(4), 575-594$.

Snow, D. W. (1965). A possible selective factor in the evolution of fruiting seasons in tropical forest. Oikos 15, 274-281.

Stiles, F. G. (1977). Coadapted competitors: The flowering seasons of hummingbird-pollinated plants in a tropical forest. Science 198, 11771178 . 
(a)

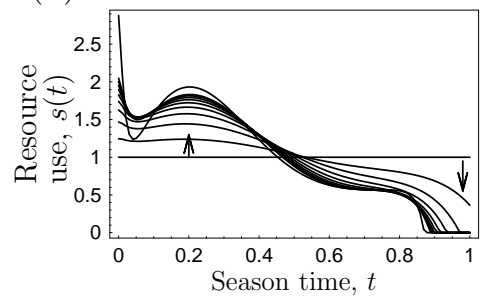

(d)

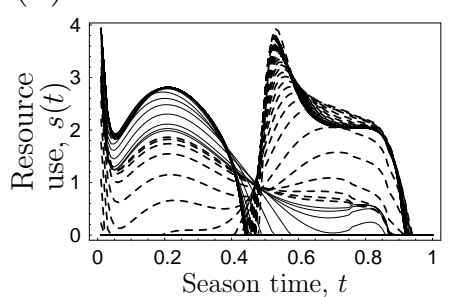

(f)

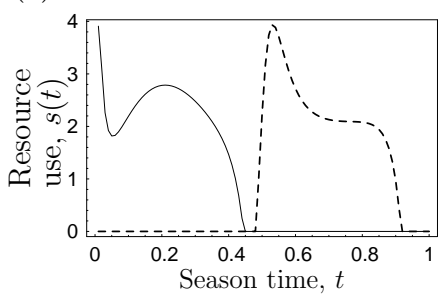

(b)

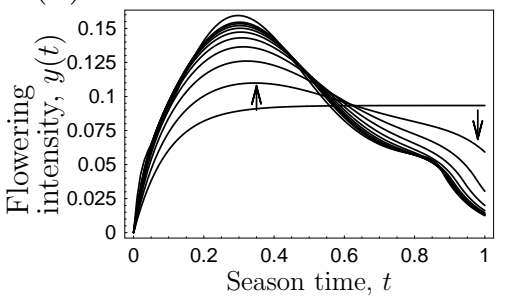

(e)

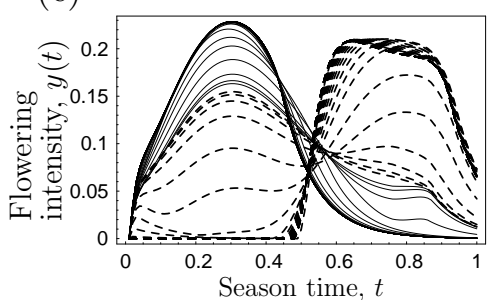

(g)

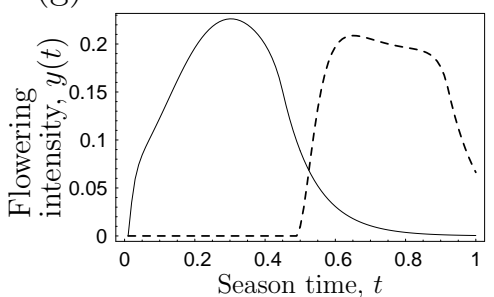

(c)

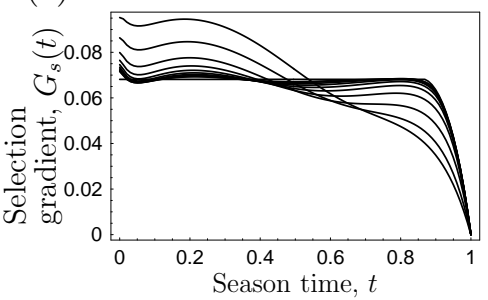

(h)

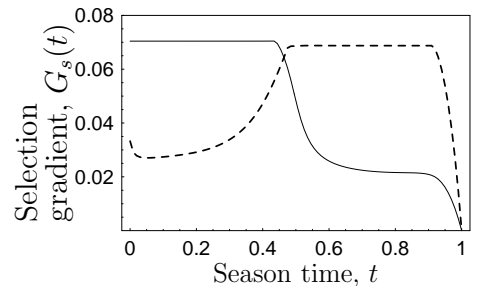

Figure 4: Monomorphic and dimorphic canonical-equation dynamics of the model (27-29), starting from a uniform allocation schedule, $s(a)=1$. (a-c) Convergence towards a monomorphic evolutionarily singular strategy that is not evolutionarily stable. (d-e) Dimorphic convergence, after evolutionary branching, towards an evolutionarily singular coalition. (f-g) Members of this coalition. (h) Corresponding selection gradients. Parameters: $\alpha=1$, $\beta=0.9, \gamma=0.2, \xi=10.51, S_{\max }=1, d=0.05$. 
(a)

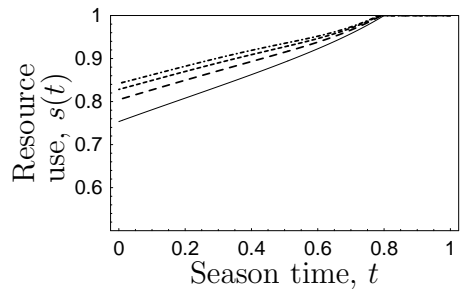

(c)

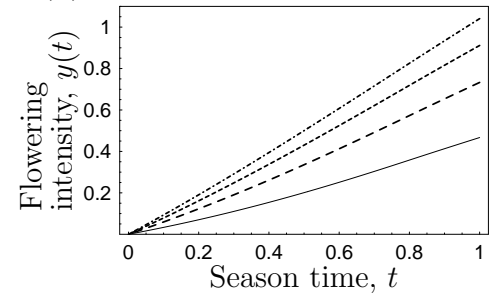

(b)

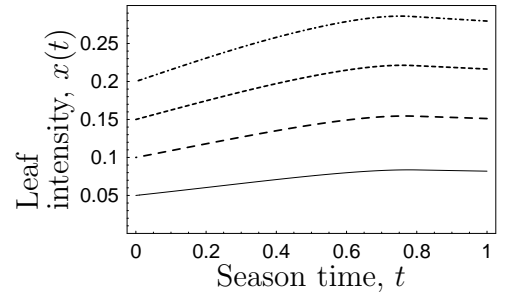

(d)

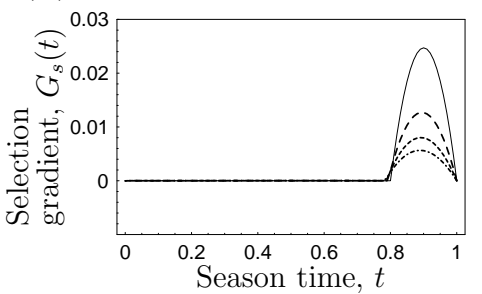

Figure 5: Evolutionarily singular strategies in the model (41) obtained with the theory of optimal control for different values of initial leaf intensity $x_{0}=0.05,0.1,0.15$, and 0.2 . (a) resource allocation strategy, $s(t)$. (b-c) Resultant leaf intensity, $x(t)$, and flowering intensity, $y(t)$. (d) Selection gradient according to equation (48). As expected, the selection gradient is 0 for $0<s(t)<1$ and positive for $s(t)=1$. Parameters: $\alpha=1, \beta=0.5, \omega=0.1$, $\xi=0.1, d=0.2, f(z)=h(z)=z /(1+0.5 x)$, and $E(t)=10$. 
(a)

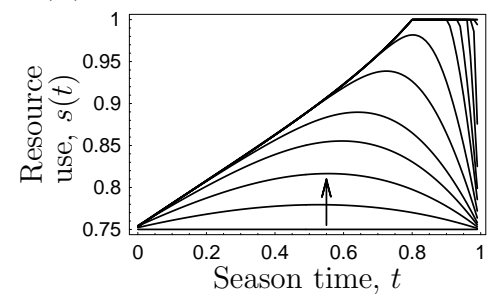

(c)

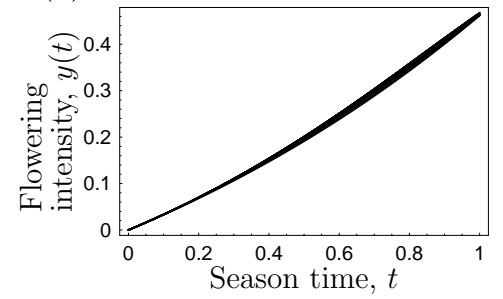

(b)

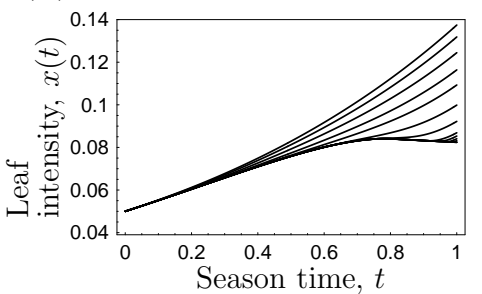

(d)

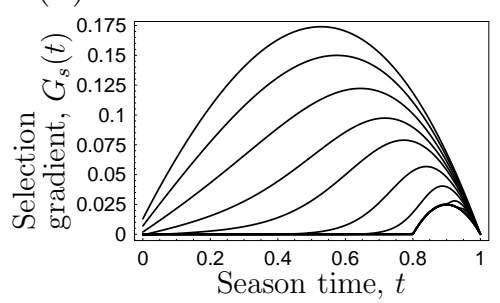

Figure 6: Canonical-equation dynamics of the model (41) starting from the uniform strategy $s(a)=0.75$. (a) Resource allocation strategy $s(t)$. (bc) Resultant leaf intensity, $x(t)$ and flowering intensity, $y(t)$. (d) Selection gradient according to equation (48). The strategy converges towards the same singular strategy as shown in Figure 5. Parameters as in Figure 5 with $x_{0}=0.05$. 